\title{
Investigation of Precipitate Refinement in Mg alloys by an Analytical Composite Failure Model
}

\author{
A. Tabei ${ }^{\mathrm{a}}$, D.S. Li ${ }^{\mathrm{b}}$, C. A. Lavender ${ }^{\mathrm{b}}$, H. Garmestani ${ }^{\mathrm{c}, *}$ \\ ${ }^{a}$ G. W. Woodruff School of Mechanical Engineering, Georgia Institute of Technology, 801 Ferst Drive NW, \\ Atlanta, GA 30332,U.S.A; tabei@gatech.edu \\ ${ }^{b}$ Pacific Northwest National Laboratory, 902 Battelle Blvd, Richland, WA 99352 \\ ${ }^{c}$ School of Materials Science and Engineering, Georgia Institute of Technology, 771 Ferst Drive NW, \\ Atlanta, GA 30332, U.S.A \\ *Corresponding Author,email address: hamid.garmestani@mse.gatech.edu
}

\begin{abstract}
An analytical model is developed to simulate precipitate refinement in second phase strengthened magnesium alloys. The model is developed based on determination of the stress fields inside elliptical precipitates embedded in a rate dependent inelastic matrix. The stress fields are utilized to determine the failure mode that governs the refinement behavior. Using an AZ31 Mg alloy as an example, the effects the applied load, aspect ratio and orientation of the particle is studied on the macroscopic failure of a single $\alpha$ $M_{17} \mathrm{Al}_{12}$ precipitate. Additionally, a temperature dependent version of the corresponding constitutive law is used to incorporate the effects of temperature. In plane strain compression, an extensional failure mode always fragments the precipitates. The critical strain rate at which the precipitates start to fail strongly depends on the orientation of the precipitate with respect to loading direction. The results show that the higher the aspect ratio is, the easier the precipitate fractures. Precipitate shape is another factor influencing the failure response. In contrast to elliptical precipitates with high aspect ratio, spherical precipitates are strongly resistant to sectioning. In pure shear loading, in addition to the extensional mode of precipitate failure, a shearing mode may get activated depending on orientation and aspect ratio of the precipitate. The effect of temperature in relation to strain rate was also verified for plane strain compression and pure shear loading cases.
\end{abstract}


Keywords: Precipitate refinement, analytical modeling, failure mode, magnesium alloys

\section{1-Introduction}

The past decade has seen rapid expansion of magnesium alloys usage in automobile and aircraft industries; mainly due to the research work on improving the mechanical behavior of light alloys. Magnesium is considered as the lightest engineering metal. Iron and Aluminum are respectively about 4.5 and 1.5 times heavier than Magnesium. Magnesium is also the eighth most common element on the earth's surface. In automobile industry, to further increase fuel efficiency, improve vehicle performance and reduce carbon dioxide emissions, more lightweight alloys have to be utilized to support vehicle weight reduction. Currently, the average usage of magnesium alloys in automobiles produced in North America is $20 \mathrm{~kg}$ per vehicle (Joost, 2012). To achieve the goal of increasing magnesium alloys usage to $100 \mathrm{~kg}$ per vehicle by 2020 , there are still several technical challenges to overcome in improving performance and manufacturability, reducing costs and implementing the modeling required, as pointed out by Joost (Joost, 2012). One of the challenges in broadening the applications of magnesium alloys is the insufficient strength and stiffness for certain structural applications. Therefore a great deal of effort has been put into strengthening magnesium alloys. The traditional high strength magnesium-based materials exploit alloying elements to refine the crystal structure by introducing ultrafine precipitate (Friedrich and Mordike, 2006; Kulekci, 2008). An example is the $\mathrm{AZ} 31 \mathrm{Mg}$ heat treatable alloy in which $\alpha-\mathrm{Mg}_{17} \mathrm{Al}_{12}$ precipitates strengthen the matrix depending on the morphology and size of the precipitates(Qiu et al., 2010). Average grain size of the matrix also depends on the precipitate size and distribution (Figueiredo and Langdon, 2010; Lin and Garmestani, 2000). Generally, smaller precipitates lead to smaller average grain size after a possible recrystallization and consequently result in enhanced mechanical responses(Li et al., 2010; Lin et al., 2000; Mikdam et al., 2009; Tabei et al., 2013). In this regards, precipitate refinement turns out to be an essential step in mechanical strengthening of magnesium alloys. 
One important microstructural phenomenon that happens in processes with severe plastic deformation is the refinement of the second phase precipitates (Backofen, 1972; Figueiredo and Langdon, 2010; Lee et al., 2000; Li et al., 2003). The usual approach towards simulating the precipitate refinement problem is through finite element analysis of damage mechanics (Eckschlager et al., 2002; Kiser et al., 1996; Li et al., 1999). These approaches are based on the tessellation of a computer generated microstructure and applying the damage criterion in each cell. Such models are computationally expensive for complicated processes such as friction stir processing, equal channel angular extrusion, high pressure torsion and so on.

In this work, we first introduce an analytical model for the prediction of brittle failure of the second phase $\alpha-M g_{17} A l_{12}$ precipitates in AZ31 Mg alloy. For the first time, closedform solutions of the stress fields inside the precipitates are derived in terms of the applied macroscopic strain rates and geometrical parameters of the second phase. The model is inspired by a fundamental investigation (Jeffery, 1922) on the motion of elliptical solid particles immersed in viscous fluids which assumes the velocity field at the matrix/second phase boundary is a linear function of position. In order to determine the mode of failure, the stress distributions were incorporated into a macroscopic failure criterion. Therefore it becomes possible to predict the failure of a single precipitate embedded in the matrix. In this regard, three cases with different loading conditions, namely plane strain compression, pure shear and simultaneous application of shear and normal strains are studied. Conventionally precipitates or inclusions are considered elliptical or ellipsoidal, mainly because of the possibility of capturing the basic geometrical and orientation features of real precipitates while mathematical description of the problem remains fairly feasible. Thus, we also assumed that the $\alpha-M g_{17} A l_{12}$ precipitates have elliptical shapes. This work focuses on well-known 2D loading conditions; namely plane strain compression and 2D pure shear as well as super position of the two. These 2D loading conditions are widely used as acceptable representations of several types of mechanical and manufacturing processes. Therefore, we believe using the "2D version" of a general 3D analytical model is appropriate and acceptable to provide the stress field in the precipitates and predict the refinement or macroscopic 
failure behavior. One important future work is to apply the full 3D version of this totally novel model to 3D loading conditions.

\section{2-Methodology}

2.1 Analytical solution for stress distribution inside an elliptical precipitate embedded in an inelastic matrix

It is assumed that the governing constitutive equation is a rate-dependent inelastic power law:

$$
\sigma_{e q}=k \dot{\varepsilon}_{e q}^{n}
$$

where $\dot{\varepsilon}_{e q}$ and $\sigma_{e q}$ are respectively the equivalent strain rate and equivalent stress. $k$ and $n$ are material constants. The model is developed based on rate-dependent constitutive laws and is applicable to materials (and temperature ranges) that can be fairly approximated with a rate-dependent law. At this stage of the modeling effort, strain hardening is ignored (with applications to hot working) but can easily be incorporated within the constitutive equations. The associated flow rule of the matrix is assumed to be as follows, with the presumption of von-Mises yield behavior:

$$
\dot{\varepsilon}_{i j}=\frac{3}{2} \frac{\dot{\varepsilon}_{e q}}{\sigma_{e q}} S_{i j}
$$

$s_{i j}$ and $\dot{\varepsilon}_{i j}$ are respectively the components of the deviatoric part of stress tensor and strain rate tensor. For convenience, stress tensor is decomposed into hydrostatic and deviatoric parts:

$$
\sigma_{i j}=\sigma^{h} \delta_{i j}+s_{i j}
$$

where $\sigma^{h}=\frac{\sigma_{m m}}{3}$ represents the hydrostatic stress and $\delta_{i j}$ is the Kronecker's delta. 
Using $\dot{\varepsilon}_{i j}=\frac{1}{2}\left(v_{i, j}+v_{j, i}\right)$, one may rewrite Eq. (3) from Eq. (1) and (2):

$$
\boldsymbol{\sigma}_{i j}=\boldsymbol{\sigma}^{h} \boldsymbol{\delta}_{i j}+\frac{k}{3} \dot{\boldsymbol{\varepsilon}}_{e q}^{n-1}\left(v_{i, j}+v_{j, i}\right)
$$

where $\dot{\boldsymbol{\varepsilon}}_{e q}=\sqrt{\frac{2}{3} \dot{\boldsymbol{\varepsilon}}_{i j} \dot{\boldsymbol{\varepsilon}}_{i j}}$.

Due to huge inelastic deformation of the mechanical processes that should result in significant precipitate refinement, elastic deformation is neglected in this analysis. Furthermore, incompressibility, $v_{k, k}=\frac{\partial v_{k}}{\partial x_{k}}=0$, is also assumed. With these assumptions and by using Eq.(1) to (4), one can simplify the equations of motion $\left(\sigma_{i j, i}=0\right)$ to:

$$
\begin{aligned}
& \boldsymbol{\sigma}_{i j, i}=\boldsymbol{\sigma}_{, i}^{h}+s_{i j, i} \\
& =\boldsymbol{\sigma}_{, i}^{h}+\frac{\partial}{\partial x_{i}}\left(\frac{2}{3} k \dot{\boldsymbol{\varepsilon}}_{e q}^{n-1} \dot{\boldsymbol{\varepsilon}}_{i j}\right) \\
& =\boldsymbol{\sigma}_{, i}^{h}+\frac{2}{3} k\left\{(n-1) \dot{\boldsymbol{\varepsilon}}_{i j} \dot{\boldsymbol{\varepsilon}}_{e q}^{n-2} \frac{d \dot{\boldsymbol{\varepsilon}}_{e q}}{d x_{i}}+\dot{\boldsymbol{\varepsilon}}_{e q}^{n-1} \dot{\boldsymbol{\varepsilon}}_{i j, i}\right\} \\
& =\boldsymbol{\sigma}_{, i}^{h}+\frac{2}{3} k\left\{(n-1) \dot{\boldsymbol{\varepsilon}}_{i j} \dot{\boldsymbol{\varepsilon}}_{e q}^{n-2} \frac{2 \dot{\boldsymbol{\varepsilon}}_{i j}}{3 \dot{\boldsymbol{\varepsilon}}_{e q}} \dot{\boldsymbol{\varepsilon}}_{i j, i}+\dot{\boldsymbol{\varepsilon}}_{e q}^{n-1} \dot{\boldsymbol{\varepsilon}}_{i j, i}\right\} \\
& =\boldsymbol{\sigma}_{, i}^{h}+\frac{2}{3} k\left\{(n-1) \dot{\boldsymbol{\varepsilon}}_{e q}^{n-1} \dot{\boldsymbol{\varepsilon}}_{i j, i}+\dot{\boldsymbol{\varepsilon}}_{e q}^{n-1} \dot{\boldsymbol{\varepsilon}}_{i j, i}\right\} \\
& =\boldsymbol{\sigma}_{, i}^{h}+\frac{2 n k}{3} \dot{\boldsymbol{\varepsilon}}_{e q}^{n-1} \dot{\boldsymbol{\varepsilon}}_{i j, i}=\boldsymbol{\sigma}_{, i}^{h}+\frac{n k}{3} \dot{\boldsymbol{\varepsilon}}_{e q}^{n-1}\left(v_{i, j i}+v_{j, i i}\right)=\boldsymbol{\sigma}_{, i}^{h}+\frac{n k}{3} \dot{\boldsymbol{\varepsilon}}_{e q}^{n-1} v_{j, i i}=0
\end{aligned}
$$

In other words:

$$
\sigma_{, i}^{h}+\eta \nabla^{2} v_{i}=0
$$

where $\boldsymbol{\eta}=\frac{n k}{3} \dot{\boldsymbol{\varepsilon}}_{e q}^{n-1}$.

In deformation analysis, the velocity gradient tensor $\mathbf{L}$ is decomposed into deformation rate $\mathbf{D}$ and spin $\mathbf{W}$ tensors:

$$
L_{i j}=D_{i j}+W_{i j}
$$


With the application of infinitesimal strain theory $\left(\dot{\varepsilon}_{i j} \approx D_{i j}=\frac{1}{2}\left(L_{i j}+L_{j i}\right)\right)$ and uniform strain rate and spin at infinity, one may write:

$$
v_{i}=\dot{\varepsilon}_{i j} x_{j}+\widehat{\varepsilon}_{i j k} w_{k} x_{j}
$$

where $v_{i}, w_{i}$ and $\widehat{\varepsilon}_{i j k}$ represent the components of velocity field, spin vectors and the permutation tensor respectively. All the strains are defined in the Eulerian framework.

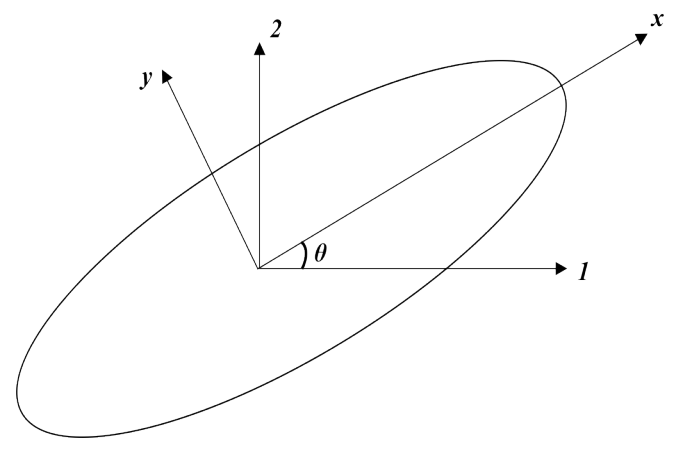

Fig. 1. The choice of corrdinate systems and the definition of particle orientation

The two coordinate systems used in this work are shown in Fig. 1. The $x-y$ precipitate coordinate system with the origin at the center of the ellipse and the bases always oriented in the direction of the semi-axes of the ellipse may rotate and displace with matrix deformation. The 1-2 system is a stationary sample coordinate system representing the direction of the externally applied loads (equivalently strain rates) to the system. The orientation angle $\theta$, between axis 1 and $\mathrm{x}$, captures the orientation of the precipitate with respect to the applied loads.

In 1922, Jeffrey (Jeffery, 1922) expanded Eq.(7) and obtained the velocity field of a continuum at the boundary of an elliptical rigid body (Refer to appendix A for more details). Similarly, here we assume that the precipitate is rigid. In other words, the elastic deformation of the precipitate is neglected compared to the inelastic deformation of its adjacent matrix material at the interface. 


$$
\begin{aligned}
& v_{1}=\left[\dot{\varepsilon}_{11}+C \gamma-2 A(\alpha+\beta)\right] x+ \\
& {\left[\dot{\varepsilon}_{12}-w_{1}+C^{\prime} \gamma+2\left(\alpha B^{\prime}-\beta B\right)\right] y-} \\
& \frac{2 x y P^{2}}{\Delta^{3}}\left[\begin{array}{l}
\left\{C^{\prime}+2\left(a^{2}+\lambda\right) B+2\left(b^{2}+\lambda\right) B^{\prime}\right\} \frac{x}{\left(a^{2}+\lambda\right)}+ \\
\left.\left\{C-2\left(a^{2}+\lambda\right) A+2\left(b^{2}+\lambda\right) A^{\prime}\right\} \frac{y}{\left(b^{2}+\lambda\right)}\right]
\end{array}\right. \\
& v_{2}=\left[\dot{\varepsilon}_{11}+w_{1}+C^{\prime} \gamma-2\left(\alpha B^{\prime}-\beta B\right)\right] x+ \\
& {\left[\dot{\varepsilon}_{12}-C \gamma-2 A^{\prime}(\alpha+\beta)\right] y-} \\
& \frac{2 x y P^{2}}{\Delta^{3}}\left[\begin{array}{l}
\left\{C^{\prime}+2\left(a^{2}+\lambda\right) B+2\left(b^{2}+\lambda\right) B^{\prime}\right\} \frac{y}{\left(b^{2}+\lambda\right)}- \\
\left\{C-2\left(a^{2}+\lambda\right) A+2\left(b^{2}+\lambda\right) A^{\prime}\right\} \frac{x}{\left(a^{2}+\lambda\right)}
\end{array}\right]
\end{aligned}
$$

Where $A, A^{\prime}, B, B^{\prime}, C$ and $C^{\prime}$ are constants. Where $\lambda$ is the positive root of

$$
\frac{x^{2}}{a^{2}+\lambda}+\frac{y^{2}}{b^{2}+\lambda}=1
$$

therefore $\lambda=0$ demarcates the boundary of the ellipse. $a$ and $b$ are respectively the major and minor semi-axes of the elliptical precipitate Other symbols are defined as follows:

$$
\begin{aligned}
& \frac{1}{P^{2}}=\frac{x^{2}}{a^{4}}+\frac{y^{2}}{b^{4}} \\
& \Delta=\sqrt{\left(a^{2}+\lambda\right)\left(b^{2}+\lambda\right)} \\
& \alpha=\int_{0}^{\infty} \frac{d \lambda}{\left(a^{2}+\lambda\right) \Delta}=\frac{2}{a(a+b)} \\
& \beta=\int_{0}^{\infty} \frac{d \lambda}{\left(b^{2}+\lambda\right) \Delta}=\frac{2}{b(a+b)} \\
& \gamma=\int_{0}^{\infty} \frac{d \lambda}{\left(a^{2}+\lambda\right)\left(b^{2}+\lambda\right) \Delta}=\frac{2}{a b(a+b)^{2}}
\end{aligned}
$$

Double differentiation of Eq. (8-a) and (8-b) and substituting into Eq. (5-b) enables one to determine the constants used in Eq. (8-a) and (8-b). For the 2D case, the boundary conditions are(Jeffery, 1922): 


$$
v_{1}=-\omega y \text { and } \quad v_{2}=\omega x
$$

where $\omega=\frac{a^{2}\left(w_{1}+\dot{\varepsilon}_{12}\right)+b^{2}\left(w_{1}-\dot{\varepsilon}_{12}\right)}{a^{2}+b^{2}}$. Therefore, one can show:

$$
\begin{aligned}
& A=\frac{\dot{\varepsilon}_{11}}{2\left((\alpha+\beta)-\left(a^{2}+b^{2}\right) \gamma\right)} \\
& A^{\prime}=-\frac{\dot{\varepsilon}_{11}}{2\left((\alpha+\beta)-\left(a^{2}+b^{2}\right) \gamma\right)} \\
& B=\frac{\alpha \dot{\varepsilon}_{12}-\gamma b^{2}\left(w_{1}-\omega\right)}{2\left(\alpha a^{2}+\beta b^{2}\right) \gamma} \\
& B^{\prime}=\frac{\beta \dot{\varepsilon}_{12}+\gamma a^{2}\left(w_{1}-\omega\right)}{2\left(\alpha a^{2}+\beta b^{2}\right) \gamma} \\
& C=\frac{\dot{\varepsilon}_{11}\left(a^{2}+b^{2}\right)}{(\alpha+\beta)-\left(a^{2}+b^{2}\right) \gamma} \\
& C^{\prime}=\frac{\dot{\varepsilon}_{12}}{\gamma}
\end{aligned}
$$

In order to determine the stress distribution inside the elliptical precipitates, closed form solutions for the traction vectors acting on the boundary of the ellipse are derived. Constants of Eq.(12) are substituted into Eq. (8-a) and (8-b) and then substituted into Eq. (4) to determine, $\sigma_{i j}$, the stress distribution right outside the boundary of the ellipse. The definition of traction vector, $T_{i}=\sigma_{i j} n_{j}$ and further simplifications leads to:

$$
\begin{aligned}
& T_{x}=P\left(L \frac{x}{a^{2}}+M \frac{y}{b^{2}}\right) \\
& T_{y}=P\left(M \frac{x}{a^{2}}+L^{\prime} \frac{y}{b^{2}}\right)
\end{aligned}
$$

n, the unit normal vector to the surface of the elliptical precipitate is:

$$
\mathbf{n}=\frac{1}{\sqrt{\frac{x^{2}}{a^{4}}+\frac{y^{2}}{b^{4}}}}\left[\begin{array}{c}
\frac{x}{a^{2}} \\
\frac{y}{b^{2}}
\end{array}\right]
$$

The definitions of the rest of symbols are as follows: 


$$
\begin{aligned}
& L=\sigma^{h}+4 \eta\left\{\frac{2}{a b}-(\alpha-\beta)\right\} A=\sigma^{h}+2 \eta(R+1) \dot{\varepsilon}_{11} \\
& M=\frac{8 \eta}{a b} B=2 \eta\left(\frac{(R+1)^{2}}{R^{2}+1}\right) \dot{\varepsilon}_{12} \\
& L^{\prime}=\sigma^{h}-4 \eta\left\{\frac{2}{a b}+(\alpha-\beta)\right\} A=\sigma^{h}-2 \eta\left(1+\frac{1}{R}\right) \dot{\varepsilon}_{11}
\end{aligned}
$$

where the aspect ratio is represented by $R=\frac{a}{b}$. Now in order to determine the stress distribution inside the elliptical particle one needs to cut a section inside the elliptical precipitate as depicted by Fig.2. A balance of force leads to:

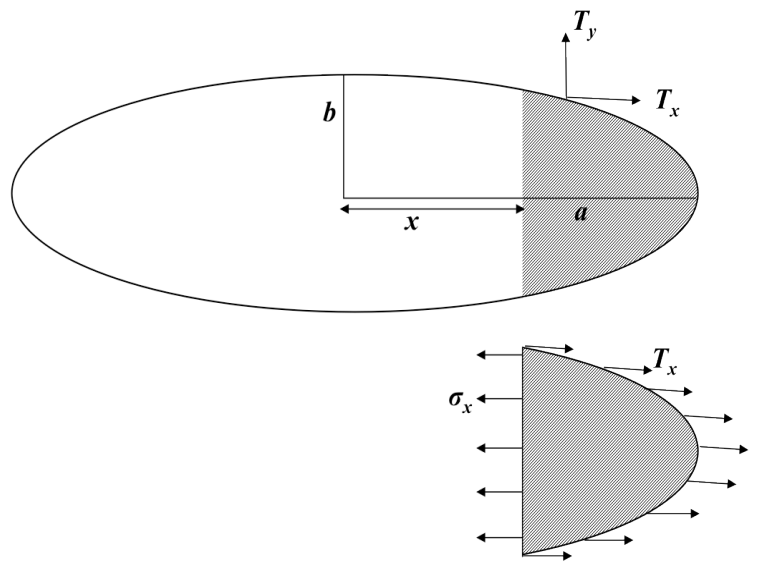

Fig. 2. Sectioning the precipitate and balancing the traction vectors with internal stresses

$$
y \sigma_{x}+\int_{x}^{a} T_{x} d s=0
$$

After substituting the arc length, $d s=\sqrt{1+\left(\frac{d y}{d x}\right)^{2}} d x$, integrating and simplification results in:

$$
\sigma_{x}=L+M R \tan \left(\frac{1}{2} \cos ^{-1}(x / a)\right)
$$


Similarly, if the section is made normal to the $y$-axis, the other component of normal stress can be determined:

$$
x \sigma_{y}+\int_{y}^{b} T_{y} d s=0
$$

Therefore:

$$
\sigma_{y}=L^{\prime}+M \frac{1}{R}\left(\frac{x}{a}+\tan \left(\cos ^{-1}(x / a)\right)\right)
$$

In a similar way, $\tau_{x y}$ can be determined:

$$
y \tau_{x y}+\int_{x}^{a} T_{y} d s=0
$$

Therefore:

$$
\tau_{x y}=M+L R \tan \left(\frac{1}{2} \cos ^{-1}(x / a)\right)
$$

Substituting Eq. (15) into (17), (19) and (21) yields the stress distribution inside an elliptical precipitate embedded in a rate dependent inelastic matrix. Eq. (17), (19) and (21) related the internal stresses at a specific position $x$ inside a 2D elliptical precipitate to the material properties $(\boldsymbol{\eta})$ and the applied load $\left(\dot{\varepsilon}_{i j}\right)$ through $L, L^{\prime}$ and $M$ coefficients as well as $R$ the aspect ratio of the precipitate.

\subsection{Macroscopic brittle failure criterion}

This work considers the dominant mechanisms for precipitate refinements and uses particle fracture as the main mechanism of refinement. Using the particle fracture in a viscous medium, the refinement phenomenon and the redistribution of the precipitates is investigated under various loading conditions. Therefore, after determining the stress field inside the precipitate, a failure criterion must be chosen to predict refinement. Clearly, any criterion, without any assumptions contrary to those mentioned in 2.1 , is applicable here theoretically. In this work, a modified 2D version of Griffith's macroscopic brittle failure criterion (Jaeger, 1970) was used to model particle refinement. Based on this model, a brittle material fails when: 


$$
\begin{aligned}
& \sigma_{1}=\sigma_{t s} \quad \text { if } \quad 3 \sigma_{1}+\sigma_{3}>0 \\
& \left(\sigma_{1}-\sigma_{3}\right)^{2}+8 \sigma_{t s}\left(\sigma_{1}+\sigma_{3}\right)=0 \quad \text { if } \quad 3 \sigma_{1}+\sigma_{3}<0
\end{aligned}
$$

where $\sigma_{1}$ and $\sigma_{3}$ are respectively the maximum principal and minimum principal stresses acting on a plane inside the material and $\sigma_{t s}$ is the tensile strength of the material. Eq. (22-a) and (22-b) respectively represents the 2D versions of mode I and mode II failures. As schematically depicted by Fig.3, mode I results in extensional separation of the pieces of the failed material while mode II is a shear type failure.

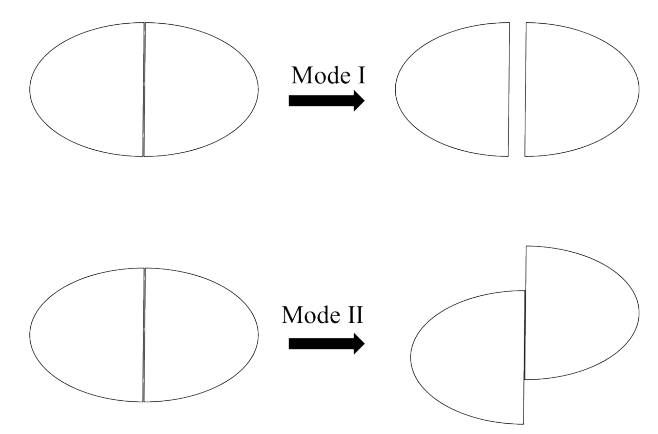

Fig. 3. Schematic illustration of the two modes of failure in the 2D version of Griffith's macroscopic brittle failure criterion

Normalizing Eq.(22-a) and (22-b) by dividing by $\sigma_{t s}$, enables one to plot a failure map for the 2D version of the Griffith's criterion of macroscopic brittle failure which is shown by Fig. 4. The failure criteria, $3 \sigma_{1}+\sigma_{3}=0$ determines which mode governs the failure behavior. Above this line in the $\sigma_{1}-\sigma_{3}$ plane, a brittle material fails under mode I and below the line, mode II controls failure. After specifying the governing mode, Eq. (22-a) and (22-b) come into play to determine if failure occurs or not. Therefore, below the condition equation and to the left of the Eq. (22-a) and (22-b) lies the stable field which is colored green in Fig. 4. Below the condition equation and to the right of Eq. (22-b) is the mode II region which is colored blue in Fig. 4. Finally, above the condition equation and to the right of Eq. (22-a) is the mode I region specified by yellow color. 


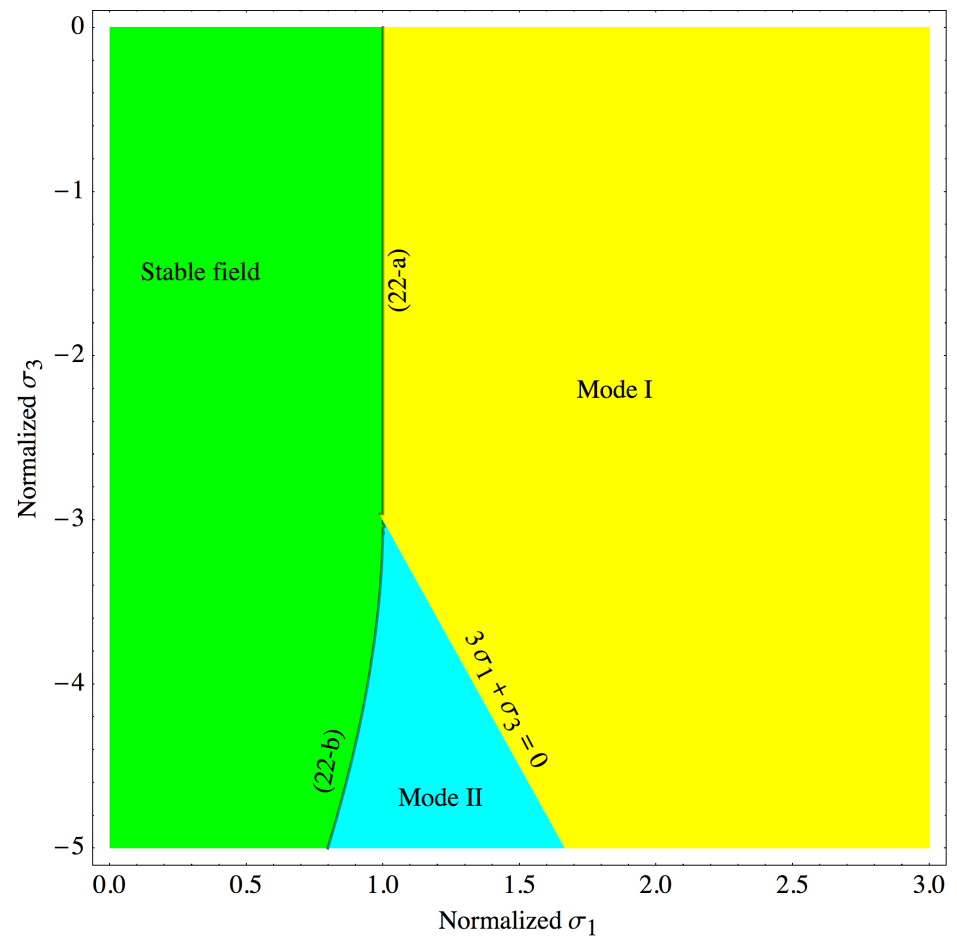

Fig. 4. General 2D failure map of the Grifith's criterion. The green, blue and yellow areas are respectively the stable field, Mode II and mode I of failure. The condition equation and the admissible portions of failure equations are labled.

Eq. (22-a) and (22-b) require the determination of the principal stresses for the purpose of failure anticipation. Eq. (17), (19) and (21) yield the full stress tensor for a 2D case (with plane stress assumption). Therefore one can compute the principal stresses: 
$\sigma_{1}=\eta\left(\sigma^{h}+\left(R-\frac{1}{R}\right) \dot{\varepsilon}_{11}+\frac{(1+R)^{2} \dot{\varepsilon}_{12}\left(a+a \sqrt{1-\frac{x^{2}}{a^{2}}}+x R^{2} \tan \left(\frac{\cos ^{-1}(x / a)}{2}\right)\right)}{R\left(1+R^{2}\right) x}\right)+$

$+\frac{1}{2} \sqrt{\frac{(1+R)^{4} \eta^{2}\left(\left(1+R^{2}\right) x \dot{\varepsilon}_{11}+a\left(1+\sqrt{1-\frac{x^{2}}{a^{2}}}\right)+x \dot{\varepsilon}_{12} R^{2} \tan \left(\frac{\cos ^{-1}(x / a)}{2}\right)\right)^{2}}{R^{2}\left(1+R^{2}\right)^{2} x^{2}}+\left(\frac{2(1+R)^{2} \eta \dot{\varepsilon}_{12}}{1+R^{2}}+\left(\sigma^{h} R-2(1+R) \dot{\varepsilon}_{11} \eta\right) \tan ^{2}\left(\frac{\cos ^{-1}(x / a)}{2}\right)\right)}$

$\sigma_{3}=\eta\left(\sigma^{h}+\left(R-\frac{1}{R}\right) \dot{\varepsilon}_{11}+\frac{(1+R)^{2} \dot{\varepsilon}_{12}\left(a+a \sqrt{1-\frac{x^{2}}{a^{2}}}+x R^{2} \tan \left(\frac{\cos ^{-1}(x / a)}{2}\right)\right)}{R\left(1+R^{2}\right) x}\right)+$

$-\frac{1}{2} \sqrt{\frac{(1+R)^{4} \eta^{2}\left(\left(1+R^{2}\right) x \dot{\varepsilon}_{11}+a\left(1+\sqrt{1-\frac{x^{2}}{a^{2}}}\right)+x \dot{\varepsilon}_{12} R^{2} \tan \left(\frac{\cos ^{-1}(x / a)}{2}\right)\right)^{2}}{R^{2}\left(1+R^{2}\right)^{2} x^{2}}+\left(\frac{2(1+R)^{2} \eta \dot{\varepsilon}_{12}}{1+R^{2}}+\left(\sigma^{h} R-2(1+R) \dot{\varepsilon}_{11} \eta\right) \tan ^{2}\left(\frac{\cos ^{-1}(x / a)}{2}\right)\right)}$

Finite element method (FEM) is applied to numerically verify the analytical results. Simulia ABAQUSTM commercial FEM code is used to perform the comparison. The assumptions underlying the analytical model were also applied to the finite element analysis. The precipitate was assumed rigid and a power-law creep behavior, as well as von-Mises plastic yielding, was imposed to the matrix. The matrix/precipitate interface is assumed to be perfect.

\subsection{AZ31 Mg alloy case studies}

To study second phase hardening due to precipitate refinement (Avedesian and Baker, 1999; Mwembela et al., 1997; Yin et al., 2005), we examine the theory developed above on AZ31 Mg alloys where precipitate size and size distribution play a remarkable role in determining the final mechanical properties of the material(Mendis et al., 2009; Mwembela et al., 1997; Yin et al., 2005). For the purpose of case studies, a single elliptical $\alpha-M g_{17} A l_{12}$ precipitate with the theoretical ultimate tensile strength of $490 \mathrm{MPa}$ for the bulk material (Ragani et al., 2011) is considered in three loading conditions: plane strain compression, pure shear and simultaneous application of tensile, compressive and 
shear strains. According to previous reports in literature, the $\alpha-M g_{17} A l_{12}$ precipitates in the AZ31 Mg alloy can form precipitates up to $40 \mu \mathrm{m}$ in size (Lapovok et al., 2008; Okayasu and Takeuchi, 2014; Wang et al., 2007; Yin et al., 2005) and can serve well for the purpose of introduction and presentation of a novel model.

The fundamental objective of the developed theory is to determine where in the precipitate, refinement occurs. In this regard, $X$ is defined as the normalized position inside the precipitate:

$$
X=\frac{x}{a}
$$

Since AZ31 is usually processed at high temperatures (Avedesian and Baker, 1999; Mwembela et al., 1997; Yin et al., 2005), (around 715K for AZ31), attention is limited to this temperature in this report. Values of $k$ and $n$ at this temperature, are respectively 49.1649 $\mathrm{MPa}$ and 0.15, which are taken from (Barnett, 2001). A temperature dependent version of the constitutive law is applied to investigate the effects of temperature (Barnett, 2001):

$$
\sigma=0.98\left(\exp \left(\frac{147000}{R T}\right)\right)^{0.15} \dot{\varepsilon}^{0.15} \quad \text { when } \quad 550 K \leq T \leq 800 K
$$

Another important aspect is the temperature dependence of tensile strength of the precipitate, $\sigma_{t s}$ is neglected in this work due to lack of literature data.

\section{3-Results and Discussion}

\subsection{Plane Strain Compression}

Fig. 5 compares the distribution of the $1^{\text {st }}$ principal stress in the precipitate obtained by analytical and finite element methods for the case of $R=2$ and $\theta=45^{\circ}$. The analytical model, predicts that the maximal value of the $1^{\text {st }}$ principal stress occurs at the center of the precipitate which is also confirmed by FEM in the example shown in Fig. 5(b). Both techniques predict that the magnitude of the maximal stress at the applied strain rate of 
$500 \mathrm{~s}^{-1}$ is around $700 \mathrm{MPa}$. In addition, the predicted stress field by FEM, in other locations in the precipitate shows good agreement compared to the analytical results.
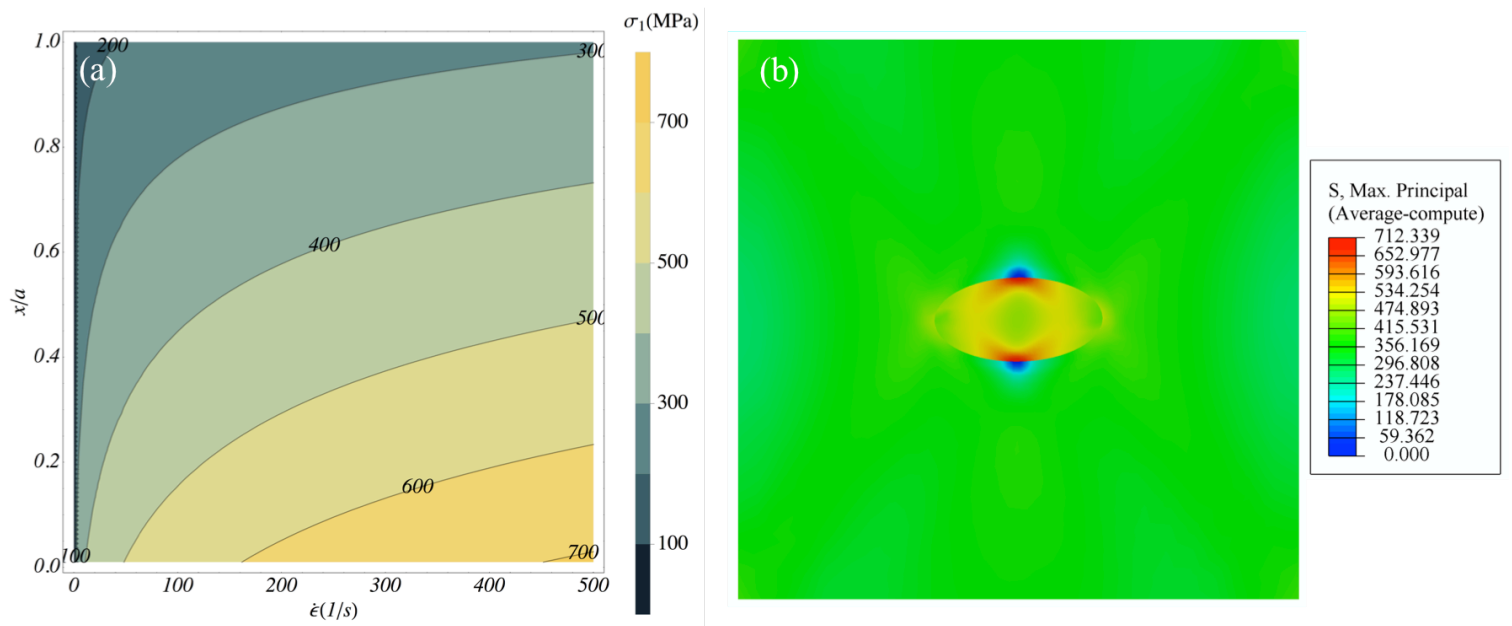

Fig. 5. (a) Constant stress contours of the $1^{\text {st }}$ principal stress induced in the precipitate as a function of the strain rate in plane strain compression. (b) FEM results for the same case at $\dot{\epsilon}=500 s^{-1}$.

An example of precipitate failure for the case of PSC of AZ31 Mg alloy (at $\mathrm{T}=715 K$ ) is shown in Fig. 6. In PSC loading at the mentioned temperature and aspect ratio, when the orientation angle of the precipitate, $\theta=0^{\circ}$, the $\alpha-M g_{17} A l_{12}$ precipitate splits around the strain rate of $\dot{\varepsilon}=39 s^{-1}$ at its center $\left(X=\frac{x}{a}=0\right)$. Increasing the strain rate makes a failure at different positions ( $X>0$ ) also possible. Interestingly, increasing the orientation angle initially reduces the strain rate at which the failure happens to values in the order of $10^{-3}$ as shown by Fig. 6(b) but for the case of $\theta=90^{\circ}$, which is the major semi-axis of the precipitate oriented along the compressive direction rather tensile, failure happens at a large strain rate, $\dot{\varepsilon}=1180 \mathrm{~s}^{-1}$. Looking at Fig. 5, one may intuitively accept that in the case of $\theta=90^{\circ}$ (major semi-axis along axis 2), mode I failure (which is the only possible mode at this type of loading) needs a significant driving force to happen. In accord with this fact, Fig. 6(c) shows the failure surface in the 3D $\dot{\varepsilon}-X-\theta$ space; below which mode I of failure happens and above, the $\alpha-M g_{17} A l_{12}$ precipitate doesn't fail. In addition, it is clear that at $\theta=90^{\circ}$ and $\dot{\varepsilon}=1180 \mathrm{~s}^{-1}$ only at $X=0$ there is the possibility of failure while if for instance, $\dot{\varepsilon}=2500 \mathrm{~s}^{-1}$ even at $\theta=90^{\circ}$, there is a possibility of particle failure even up to 
$X \approx 0.18$. According to theses results, in PSC, always mode I governs the macroscopic failure response.
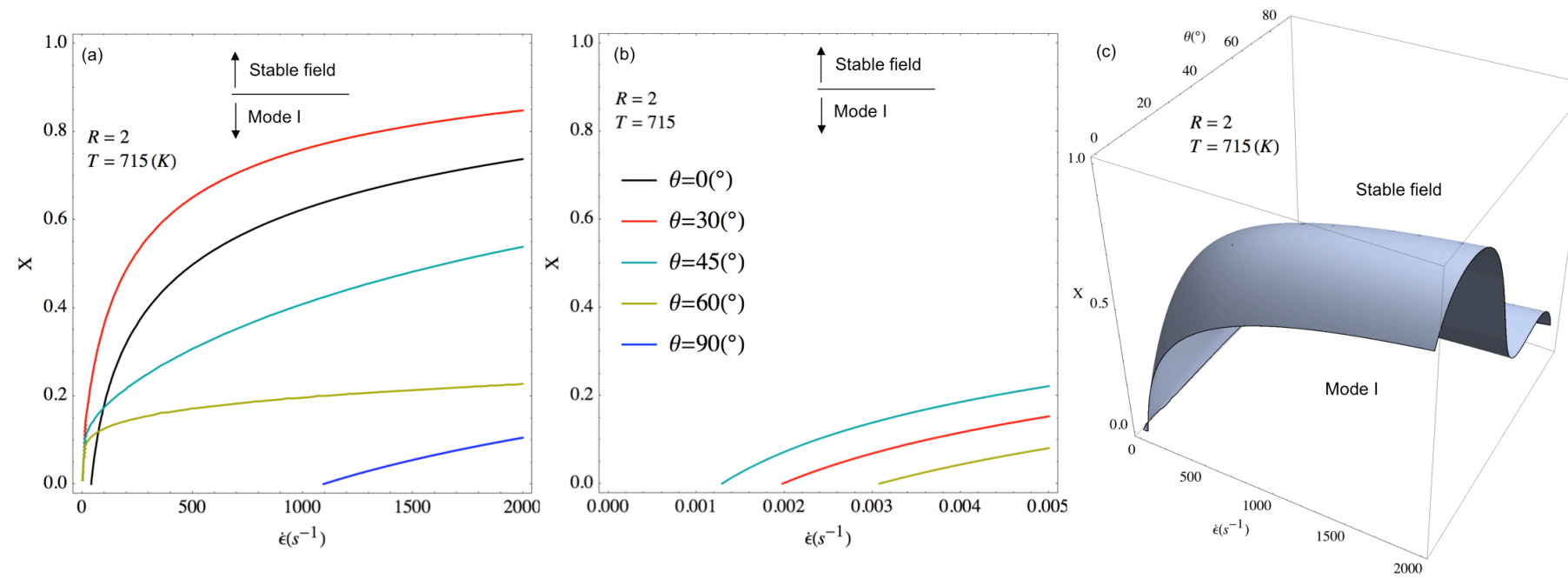

Fig. 6. Precipitate failure map for hot PSC of AZ31 Mg alloy. (a) and (b) show the horizontal axis at different scales. The inset legend in (b) also applies to(a). In both (a) and (b), above the curves is the stable field and below the curves, mode I occurs. (c) shows the 3D failure surface in the strain rateorientation-position space. Below the surface, mode I happens while above the surface is the stable field.

As shown by Eq. (23-a) and (23-b), another important morphological feature affecting the refinement behavior is the precipitate aspect ratio. Fig. 7(a) illustrates the precipitate failure map for different aspect ratios. At $\theta=0^{\circ}$ and $T=715 \mathrm{~K}$, when the applied strain rate exceeds $39 \mathrm{~s}^{-1}$, the precipitate with $R=2$ fails while a precipitate with $R=3$ fails at strain rates in the order of $10^{-2}$ and at with $R=4$ and 5 , the precipitate fails at rate in the order of $10^{-3}$. According to Fig. 7(a), the higher the aspect ratio is, the easier the precipitate splitting is and the range of position inside the precipitate that failure can happen becomes wider.

Fig. 7(b) depicts the high resistance to failure in circular precipitates $(R=1)$ where a strain rate close to $1.1 \times 10^{4}\left(\mathrm{~s}^{-1}\right)$ is required to split the precipitate. In other words, increasing the applied strain rates makes refinement of precipitate with lower aspect ratio possible as shown by Fig. 7(c). According to Fig. 7(c), at $\theta=0^{\circ}$ and $T=715 K$, it is impossible to refine the precipitates with $R<2$ at $\dot{\varepsilon}=40 \mathrm{~s}^{-1}$ but if the strain rate increases to $5000 \mathrm{~s}^{-1}$, refinement of precipitates with $R \approx 1.2$ becomes possible. 

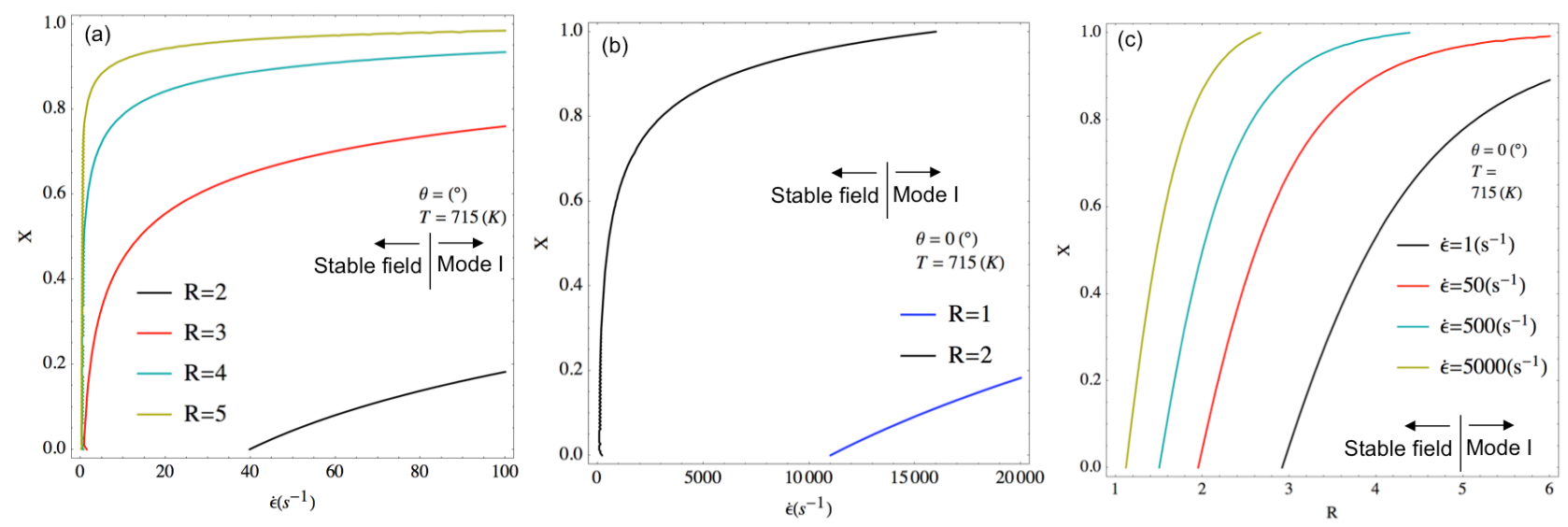

Fig. 7. (a) Effect of aspect ratio on precipitate failure. (b) High refinement resistance of circular (c) Effects of strain rate on precipitate failure

\subsection{Pure Shear}

Fig. 8 illustrates the constant stress contours of the $1^{\text {st }}$ principal stress in the precipitate obtained by analytical and finite element methods for the case of $R=2$ and $\theta=45^{\circ}$ in pure shear loading. Both techniques predict that a maximal stress value around $400 \mathrm{MPa}$ at the shear rate of $500 \mathrm{~s}^{-1}$ as well as an acceptable stress distribution of stress in other locations in the precipitate.
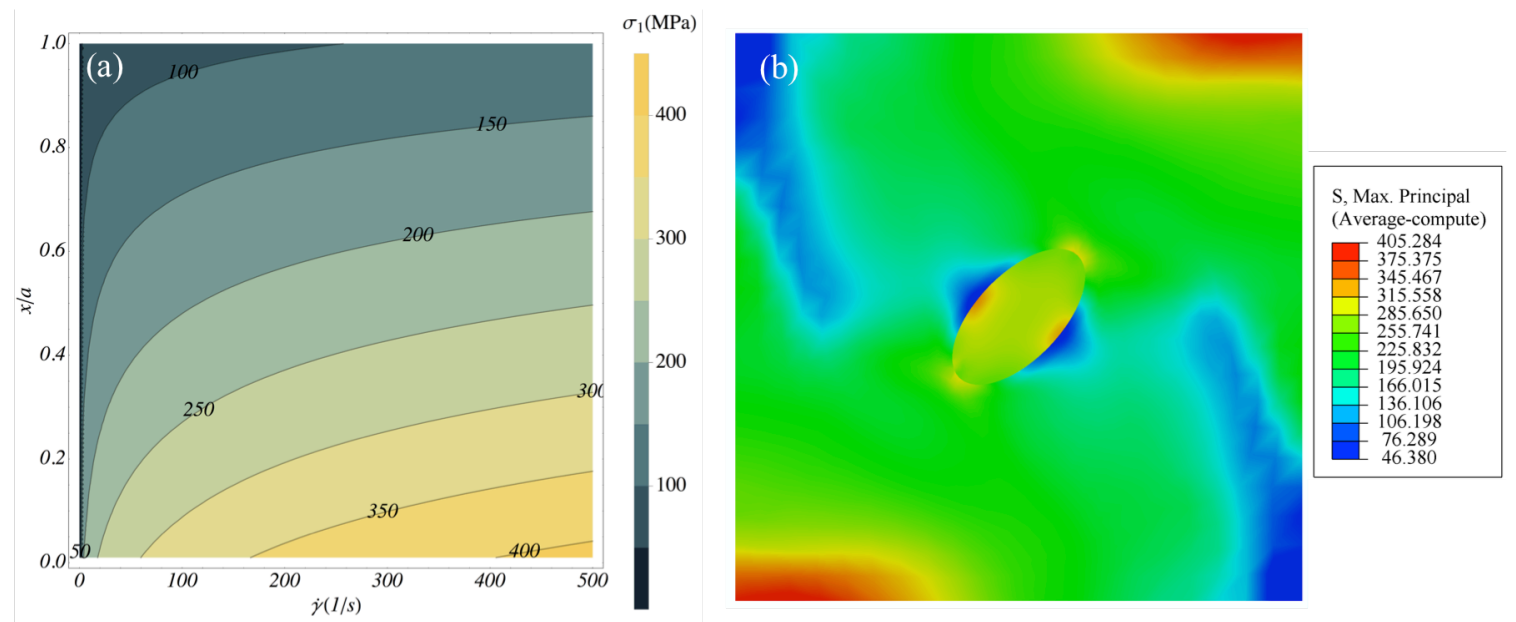

Fig. 8. Fig. 9. (a) Constant stress contours of the $1^{\text {st }}$ principal stress induced in the precipitate as a function of the strain rate in pure shear. (b) FEM results for the same case at $\dot{\gamma}=500 \mathrm{~s}^{-1}$. 

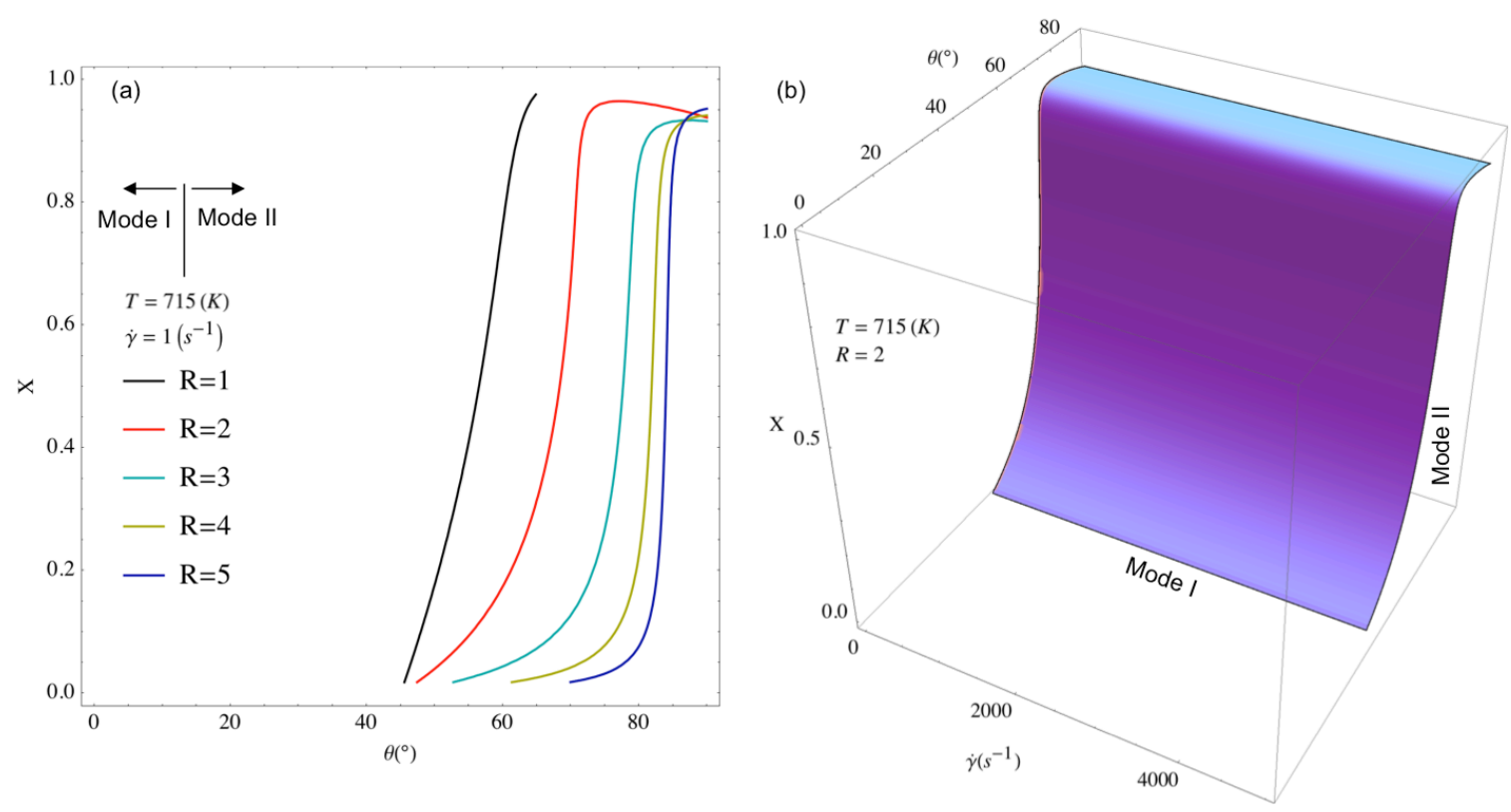

Fig. 10. Failure mode thresholds in pure shear loading. (a) Threshold curve in a 2D processing plane of $X$ and $\boldsymbol{\theta}$ with different aspect ratios. Mode I and II are at the left and right side of the threshold curves, respectively. (b) Threshold plane in a processing space of shear rate $\dot{\gamma}, \mathbf{X}$ and $\theta$.

Following the procedure explained in section 2, one can conclude that in pure shear loading, there is the possibility of occurrence of the two failure modes. In Fig. 9(a), the left hand side of the threshold curves is the region governed by mode I of failure while the left side is governed by mode Fig. 9(a) is the plot of the condition equation in Eq. (22) and determines what mode of failure controls the refinement behavior. At this point, the question whether failure happens or not still remains open. Clearly, failing depends on the applied strain rate but mode of failure is only dictated by the loading condition therefore as shown by Fig. 9(b), strain rate doesn't affect the mode of failure. According to Fig. 9(a) the mode $\mathrm{I} /$ mode II transition occurs at $\theta=45^{\circ}$ when $X=0$ and $R=1$. This transition happens at higher orientation angles if the precipitate is going to fail at positions away from the origin of the ellipse. Furthermore, precipitate with higher asymmetry, or larger aspect ratio, will have higher possibility for mode I failure due to the threshold curve moving toward the mode II zone. In other words, the orientation angle at which transition happens at $X=0$ also moves towards higher angles. 

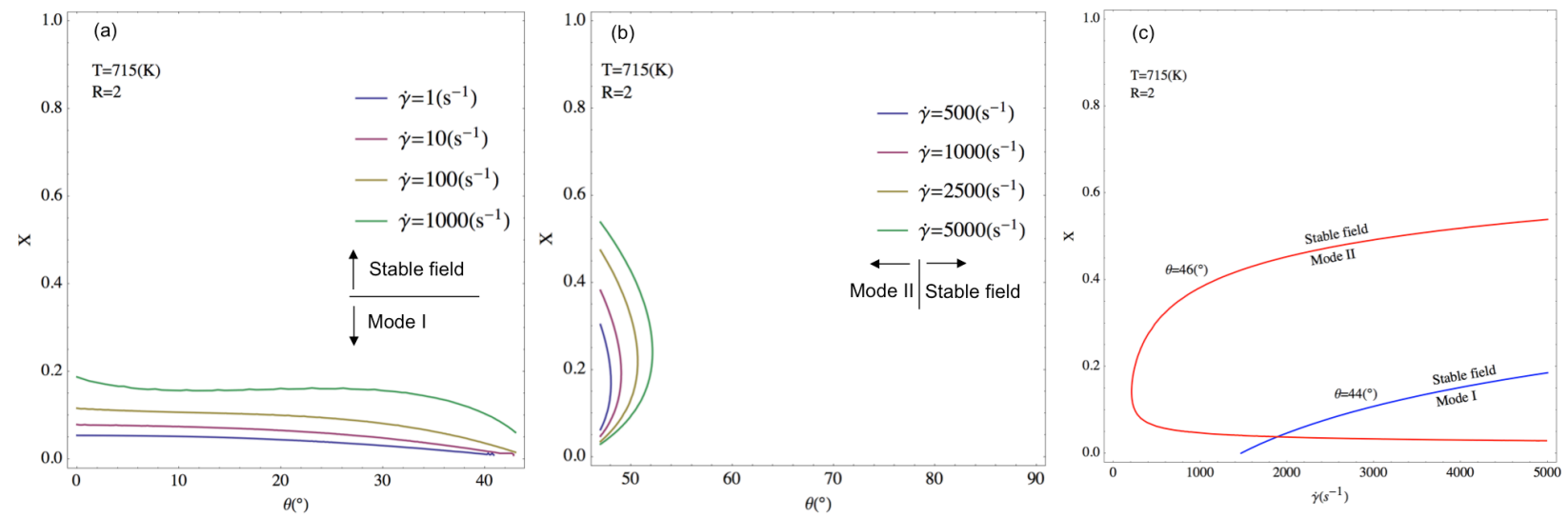

Fig. 11. Pure shear failure response in the orientation range of (a) mode I and (b) mode II. (c) Strain rate dependence at the mode I-II transition orientation

Fig.10(a) shows that failure can occurs mostly at the center of the precipitate when $\theta \leq$ $45^{\circ}$. Increasing the shear rate, increases the position range where failure can occur; i.e. making failure at positions other than the center also possible. Fig. 10(b) shows the same plots for $\theta \geq 45^{\circ}$, where mode II governs the refinement behavior. (Refer to Fig. 9(a) for occurrence of each mode of failure in pure shear). Again, as the applied shear rate increases, the range of positions at which failure can happen increases from the center of the precipitate towards the apex.
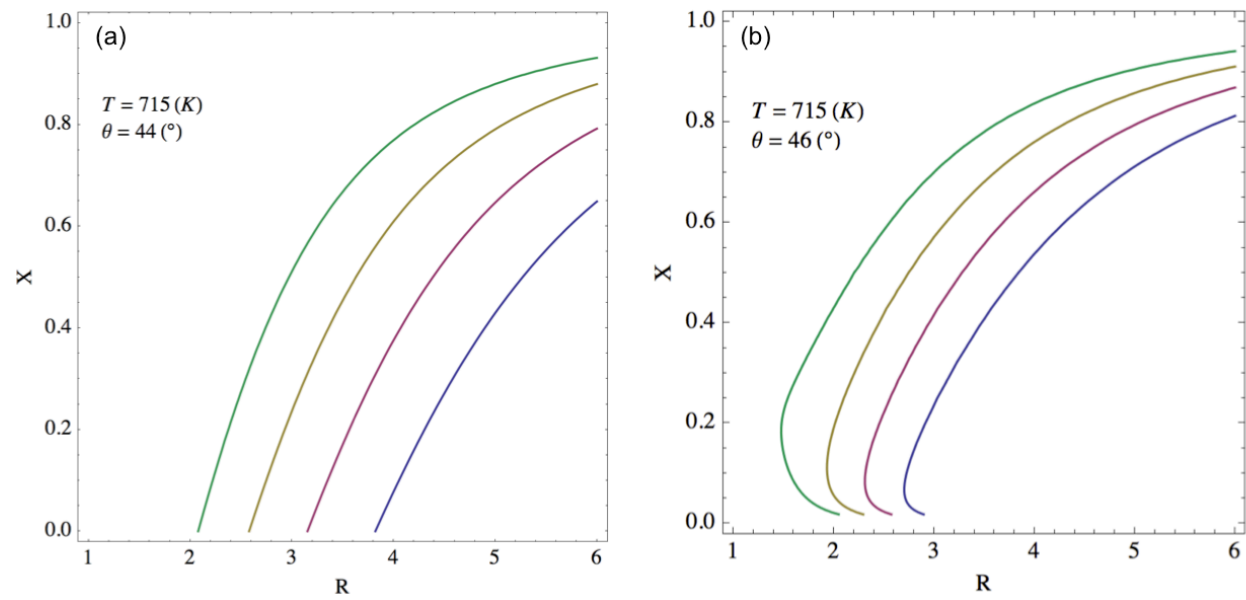

$$
\begin{aligned}
& -\dot{\gamma}=1\left(\mathrm{~S}^{-1}\right) \\
& -\dot{\gamma}=10\left(\mathrm{~S}^{-1}\right) \\
& -\dot{\gamma}=100\left(\mathrm{~S}^{-1}\right) \\
& -\dot{\gamma}=1000\left(\mathrm{~S}^{-1}\right)
\end{aligned}
$$

Fig. 12. Effects of aspect ratio in pure shear for (a) mode I and (b) mode II of failure 
According to Fig. 9(a), for a specific aspect ratio and orientation $\left(\theta \geq 45^{\circ}\right)$, the mode II region constricts as the position gets closer to the apex of the precipitate; i.e. the curves in Fig. 9(a) all have positive curvature. This is also shown in Fig. 10(b); where the range of positions and orientations at which refinement may occur is relatively small; e.g. at very high strain rate of $5000\left(\mathrm{~s}^{-1}\right)$, failure only possible at the range $45^{\circ} \leq \theta \leq 52^{\circ}$ and $0 \leq X \leq 0.55$. Furthermore, Fig. 10(b) shows that at lower shear rates, mode II region doesn't start at the center of the precipitate (i.e. $X=0$ ).

Fig. 10(c) illustrates the response of the precipitate very close to the transition angle, $\theta=$ $45^{\circ}$ for $R=2$. At the left side of the transition $\left(\theta=44^{\circ}\right)$, where mode I governs the failure, refinement occurs at much higher shear rates $\left(\dot{\gamma} \approx 1500 s^{-1}\right)$ compared to lower angles shown in Fig. 10(a). For the right side of the transition $\left(\theta=46^{\circ}\right)$, the behavior is similar to higher angles. More importantly, in mode II, precipitate failure does not occur that center of the ellipsoid opposite to mode I failure. Increasing the applied shear rate asymptotically expands the mode II region towards $X=0$.

Fig. 11 exhibits the effects of aspect ratio in the case of PS. Similar to PSC, higher aspect ratios ease the failure of the precipitates. Or equivalently, higher strain rates make cleaving more circular precipitates possible. The effect of aspect ratio is the same on mode I and mode II of failure as shown by Fig. 11(a) and (b) respectively.

\subsection{Application of Simultaneous Tensile, Compressive and Shear (STCS) strains}

Simultaneous applying of independent shear and normal strains to the bulk material is the next studied case. The normal strain rates $\dot{\varepsilon}$, consist of tensile and compressive strains with the same magnitude applied along axes 1 and 2 respectively. An independent shear strain rate $\dot{\gamma}$, is superimposed to apply both loads at the same time.

Fig. 12(a) shows the failure mode condition (Eq. (22)) check for simultaneous tensile, compressive and shear (STCS) case. According to Fig. 12(a), the governing mode does not depend on the individual strain magnitudes; but the ratio of shear and normal strain rates controls the behavior. This is not evident from the derived equations and is a fact observable when comparing the results. The ratio of applied strains is defined as $\rho$ :

$$
\rho=\frac{\dot{\gamma}}{\dot{\varepsilon}}
$$


According to Fig. 12(a), when $\rho$ goes to zero, the material failure behavior moves towards PSC while increasing the strain rates ratio to larger values results in shear behavior domination which makes occurrence of mode II also possible. STCT failure maps are shown in Fig. 12(b), (c) and (d).

Comparing to PSC, one realizes that there is a strong synergetic effect when both shear and normal strains are applied. Fig. 12(b) shows that at $R=2$ and $\theta=0^{\circ}$ mode I of failure can split the precipitate at $X=0$ while $\dot{\gamma}=0.001 \mathrm{~s}^{-1}$ and $\dot{\varepsilon}$ is in the order of $10^{-3} \mathrm{~s}^{-1}$. Additionally, on the conditions that mode I dominates the precipitates refinement, increasing the shear rate expands the failure region at a constant normal strain rate, as shown by Fig. 12(b).

Fig. 12(c) illustrates that at comparatively low strain rates of $\dot{\varepsilon}=0.1 \mathrm{~s}^{-1}$ and $\dot{\gamma}=0.001 \mathrm{~s}^{-1}$, mode I occurs in all the possible ranges of orientation. At $\dot{\gamma}=0.1\left(s^{-1}\right)$ and $\dot{\varepsilon}$ in the order of $10^{-3}\left(\mathrm{~s}^{-1}\right)$, mode II may occur at all the range of position $(0 \leq X \leq 1)$ with the orientation ranging from $45^{\circ}$ to $50^{\circ}$. Fig. 12(e) shows the effect of aspect ratio in STCS loading. In trend, similar to PSC and PS but much more intensely, increasing the aspect ratio increases the possibility of refinement. Interestingly, in STCS increasing the aspect ratio from 2 to higher values initially reduces the range at which failure may occur, but more increase results in the expansion of the failure region again towards the apexes of the ellipse. In addition, higher aspect ratios increase the orientations range at which refinement may happen. 

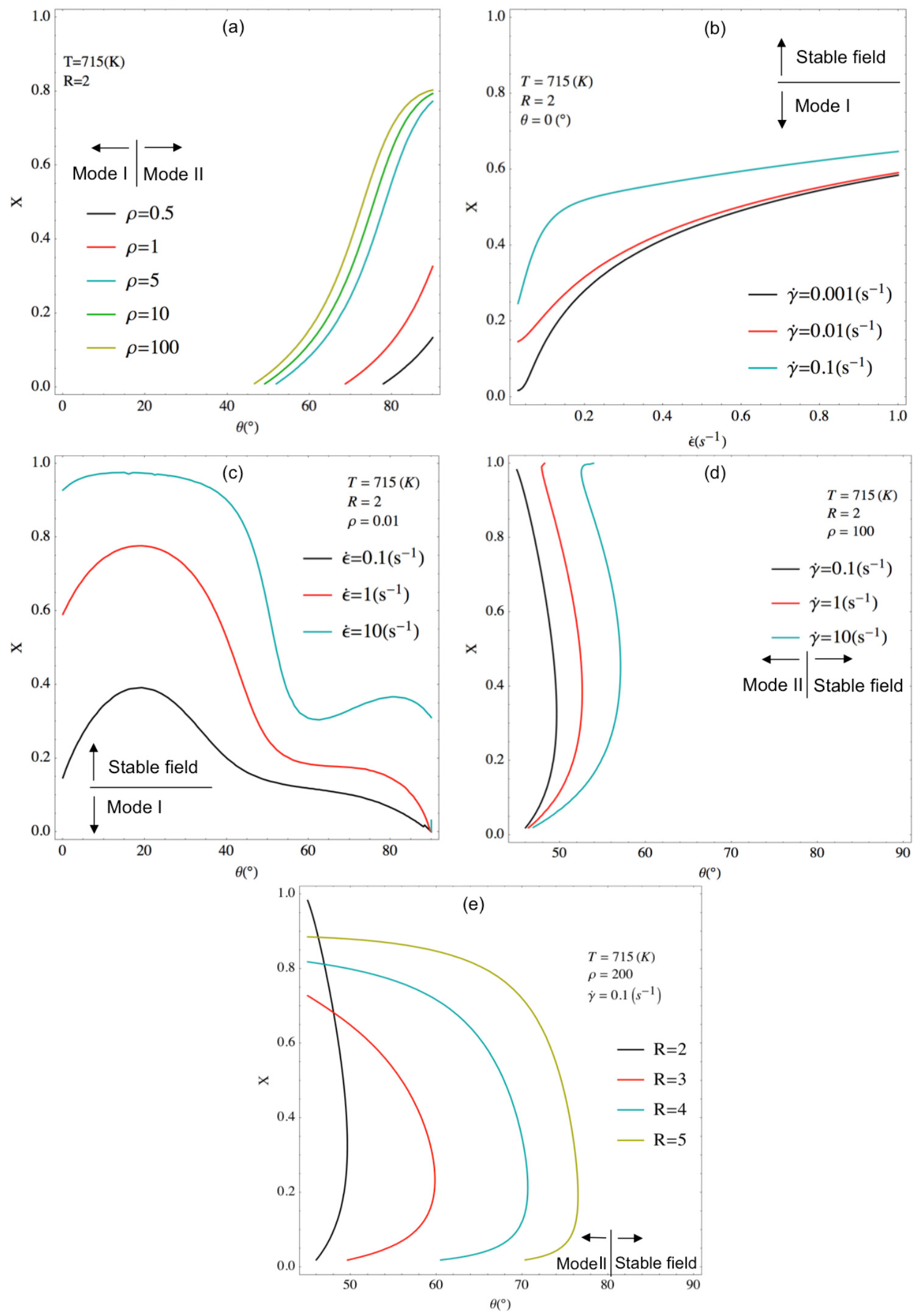
Fig. 12. (a) Failure mode threshold in STCS. (b) STCS mode I failure map as a function of normal strain rate. (c) STCT mode I failure map as a function of orientation. (d) STCT mode II failure map as a function of orientation. (e) Effect of aspect ratio on mode I in STCT

\subsection{Temperature effects}
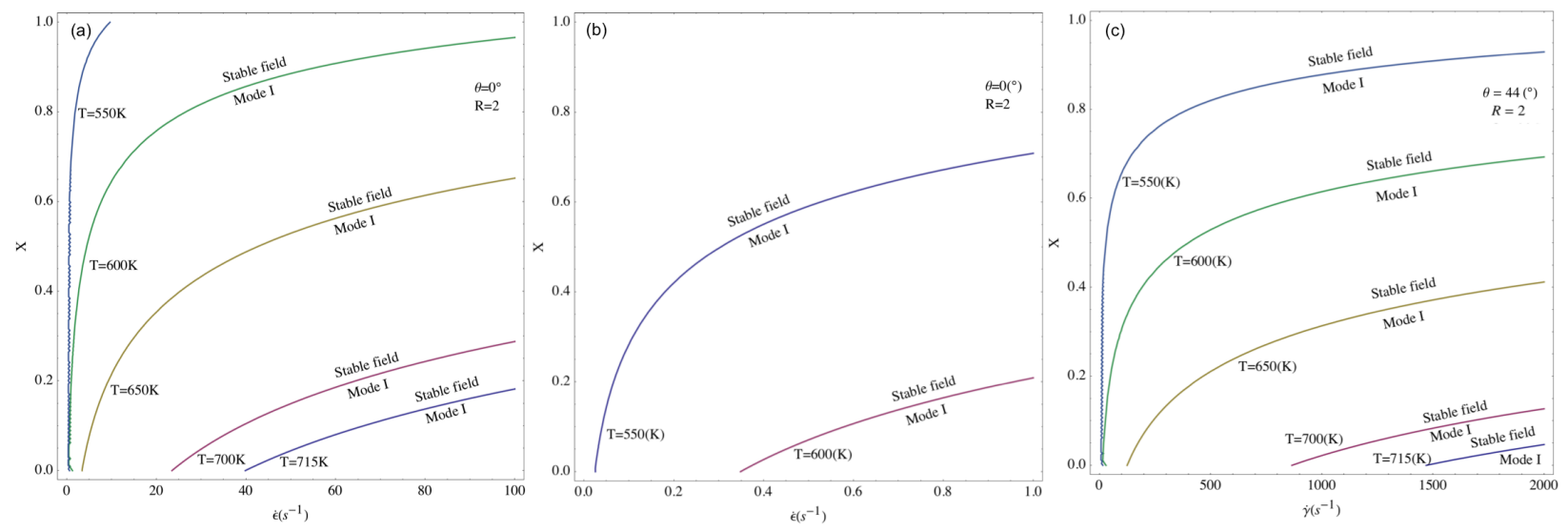

Fig. 13. Effect of temperature on failure behavior in (a) \&(b) plane strain compression and (c) pure shear

To consider the temperature effects, a temperature-dependent constitutive law (Eq. (25)) is incorporated here instead of its isothermal form (Eq. (1)). Fig. 13 depicts three failure maps in PSC and PS plotted with the assumptions made in section 3.4. As expected, reducing temperature acts similar to increasing strain rate. According to Fig. 13(a), at 550 $K$, the minimum allowed temperature by Eq. (25), failure may occur at a large position range from center to regions close to the apex of the precipitate (failure at apex physically means no refinement). Fig. 13(b) zooms in the left corner of Fig. 13(a) to better demonstration of the effect of temperature. When the temperature drops from 600 to 550 $K$, the strain rate required for precipitate refinement is reduced by an order of magnitude. Fig. 13(c) shows a similar temperature effect in pure shear as that in plane strain compression. Fig. 13 demonstrates that precipitate refinement at higher temperature is much more difficult comparing with lower temperatures which provides guidance in precipitate refinement during thermomechanical processes. In other words, precipitate 
refinement should be performed by thermomechanical processing at low temperature instead of high temperature processes such as hot rolling or hot torsion compression.

\section{4-Conclusions}

Stress fields inside a 2D elliptical precipitate is expressed in terms of the applied loading conditions, precipitate aspect ratio $R$, and orientation $\theta$ to the loading direction. In plane strain compression, the extensional mode of failure always occurs while the applied strain rate at which precipitate splitting happens strongly depends on the orientation. In addition, precipitates with low aspect ratios have higher resistance to failure. In pure shear, depending on the orientation and aspect ratio of the precipitate, there is a possibility of refinement under the shearing mode of failure in addition to the extensional mode. When the extensional mode occurs, increasing the strain rate expands the fragmentation position range inside the precipitate while under the shearing mode, depending on the shear rate, there is a much smaller position range that failure may occur. Similar to plane strain compression, larger precipitates break much more easily compared to smaller ones. In simultaneous application of shear and normal strains, the ratio of shear to normal strains determine the behavior meaning that, at high shear strains and low normal strains the behavior tends towards pure shear and vice versa. However, the normal strain behavior still dominates the whole response and compared to pure shear or plane strain compression, splitting occurs at much lower strain rates. Finally, the effects of increasing the temperature of loading on the macroscopic failure of the precipitate were discussed.

\section{Acknowledgements}

Authors of this paper acknowledge the source of funding for this effort: the Office of Vehicle Technology (OVT) of the Department of Energy's Office of Energy Efficiency and Renewable Energy through PNNL's operating contract DE-AC05-76RL01830. Specifically, Mr. Will Joost of the OVT has been instrumental in providing guidance for the project activities. 


\section{References:}

Avedesian, M.M., Baker, H., 1999. ASM specialty handbook: magnesium and magnesium alloys. ASM international 274.

Backofen, W.A., 1972. Deformation processing. Addison-Wesley Reading, MA.

Barnett, M., 2001. Influence of deformation conditions and texture on the high temperature flow stress of magnesium AZ31. Journal of light metals 1, 167-177. Eckschlager, A., Han, W., Böhm, H.J., 2002. A unit cell model for brittle fracture of particles embedded in a ductile matrix. Computational materials science 25, 85-91. Figueiredo, R.B., Langdon, T.G., 2010. Grain refinement and mechanical behavior of a magnesium alloy processed by ECAP. Journal of materials science 45, 4827-4836. Friedrich, H.E., Mordike, B.L., 2006. Magnesium technology: metallurgy, design data, automotive applications. Springer.

Jaeger, J.C., 1970. Elasticity, fracture and flow: with engineering and geological applications. Barnes \& Noble.

Jeffery, G.B., 1922. The motion of ellipsoidal particles immersed in a viscous fluid. Proceedings of the Royal Society of London. Series A, Containing Papers of a Mathematical and Physical Character 102, 161-179.

Joost, W.J., 2012. Reducing Vehicle Weight and Improving US Energy Efficiency Using Integrated Computational Materials Engineering. JOM 64, 1032-1038.

Kiser, M., Zok, F., Wilkinson, D., 1996. Plastic flow and fracture of a particulate metal matrix composite. Acta materialia 44, 3465-3476.

Kulekci, M.K., 2008. Magnesium and its alloys applications in automotive industry. The International Journal of Advanced Manufacturing Technology 39, 851-865.

Lapovok, R., Estrin, Y., Popov, M., Rundell, S., Williams, T., 2008. Enhanced superplasticity of magnesium alloy AZ31 obtained through equal-channel angular pressing with back-pressure. Journal of Materials Science 43, 7372-7378.

Lee, Y.C., Dahle, A.K., StJohn, D.H., 2000. The role of solute in grain refinement of magnesium. Metallurgical and Materials Transactions A 31, 2895-2906.

Li, D., Baniassadi, M., Garmestani, H., Ahzi, S., Reda Taha, M., Ruch, D., 2010. 3D reconstruction of carbon nanotube composite microstructure using correlation functions. Journal of Computational and Theoretical Nanoscience 7, 1462-1468.

Li, D., Garmestani, H., Schoenfeld, S., 2003. Evolution of crystal orientation distribution coefficients during plastic deformation. Scripta materialia 49, 867-872.

Li, M., Ghosh, S., Richmond, O., 1999. An experimental-computational approach to the investigation of damage evolution in discontinuously reinforced aluminum matrix composite. Acta materialia 47, 3515-3532.

Lin, S., Garmestani, H., 2000. Statistical continuum mechanics analysis of an elastic twoisotropic-phase composite material. Composites Part B: Engineering 31, 39-46.

Lin, S., Garmestani, H., Adams, B., 2000. The evolution of probability functions in an inelasticly deforming two-phase medium. International journal of solids and structures 37, 423-434.

Mendis, C.L., Oh-Ishi, K., Kawamura, Y., Honma, T., Kamado, S., Hono, K., 2009. Precipitation-hardenable Mg-2.4 Zn-0.1 Ag-0.1 Ca-0.16 Zr (at.\%) wrought magnesium alloy. Acta Materialia 57, 749-760. 
Mikdam, A., Makradi, A., Ahzi, S., Garmestani, H., Li, D.S., Remond, Y., 2009.

Effective conductivity in isotropic heterogeneous media using a strong-contrast statistical continuum theory. Journal of the Mechanics and Physics of Solids 57, 76-86.

Mwembela, A., Konopleva, E.B., McQueen, H.J., 1997. Microstructural development in Mg alloy AZ31 during hot working. Scripta Materialia 37, 1789-1795.

Okayasu, M., Takeuchi, S., 2014. Mechanical strength and failure characteristics of cast $\mathrm{Mg}-9 \% \mathrm{Al}-1 \% \mathrm{Zn}$ alloys produced by a heated-mold continuous casting process: Fatigue properties. Materials Science and Engineering: A 600, 211-220.

Qiu, W., Han, E.-H., Liu, L., 2010. Effect of heat treatment on microstructures and mechanical properties of extruded-rolled AZ31 Mg alloys. Transactions of Nonferrous Metals Society of China 20, s481-s487.

Ragani, J., Donnadieu, P., Tassin, C., Blandin, J., 2011. High-temperature deformation of the $\gamma$-Mg $<$ sub $>17</$ sub $>\mathrm{Al}<\mathrm{sub}>12</$ sub $>$ complex metallic alloy. Scripta Materialia 65, 253-256.

Tabei, S., Sheidaei, A., Baniassadi, M., Pourboghrat, F., Garmestani, H., 2013. Microstructure Reconstruction and Homogenization of Porous Ni-YSZ Composites for Temperature Dependent Properties. Journal of Power Sources. Wang, Y., Zeng, X., Ding, W., Luo, A.A., Sachdev, A.K., 2007. Grain refinement of AZ31 magnesium alloy by titanium and low-frequency electromagnetic casting. Metallurgical and Materials Transactions A 38, 1358-1366.

Yin, D.L., Zhang, K.F., Wang, G.F., Han, W.B., 2005. Warm deformation behavior of hot-rolled AZ31 Mg alloy. Materials Science and Engineering: A 392, 320-325.

\section{Appendix A}

In addition to the auxiliary parameters defined in Eq. (10), we need to define:

$$
\begin{aligned}
& \Omega=\int_{\lambda}^{\infty}\left(\frac{x^{2}}{a^{2}+\lambda}+\frac{y^{2}}{b^{2}+\lambda}-1\right) \frac{d \lambda}{\Delta} \\
& \chi=\gamma x y
\end{aligned}
$$


where $\lambda$ and $\Delta$ have the meanings defined earlier. $\gamma$ is also defined in Eq. (10) (not to be mistaken with shear strain). Clearly, $\Omega$ gives the solution to Laplace's equation in $2 \mathrm{D}$ confocal ellipsoidal coordinates. We seek the solution to Eq. (5-b) in terms of the first and second differentials of $\Omega$ and first differentials of $\chi$. From Eq. (9) one may write:

$$
\begin{aligned}
& \frac{\partial \lambda}{\partial x}=\frac{2 x \Pi^{2}}{a^{2}+\lambda} \\
& \frac{\partial \lambda}{\partial y}=\frac{2 y \Pi^{2}}{b^{2}+\lambda}
\end{aligned}
$$

where:

$$
\frac{1}{\Pi^{2}}=\frac{x^{2}}{\left(a^{2}+\lambda\right)^{2}}+\frac{y^{2}}{\left(b^{2}+\lambda\right)^{2}}
$$

At the boundary of the ellipse $(\lambda=0), \Pi=P$ where $P$ is defined in Eq. (10). Also:

$$
\begin{aligned}
& \frac{\partial \Omega}{\partial x}=2 \alpha x \\
& \frac{\partial^{2} \Omega}{\partial x^{2}}=2 \alpha-\frac{4 x^{2} \Pi^{2}}{\left(a^{2}+\lambda\right)^{2} \Delta}
\end{aligned}
$$

Differentials of Eq. (A-4) can be similarly written with respect to $y$. The velocity field can be written in the following form (Jeffery, 1922):

$$
\begin{aligned}
& v_{1}=v_{1}^{\infty}+\frac{\partial}{\partial x}\left(C^{\prime} \chi\right)+\frac{\partial}{\partial y}(C \chi)+A\left(x \frac{\partial^{2} \Omega}{\partial x^{2}}-\frac{\partial \Omega}{\partial x}\right)+ \\
& B\left(x \frac{\partial^{2} \Omega}{\partial x \partial y}-\frac{\partial \Omega}{\partial y}\right)+y\left(A^{\prime} \frac{\partial^{2} \Omega}{\partial x \partial y}+B^{\prime} \frac{\partial^{2} \Omega}{\partial x^{2}}\right) \\
& v_{2}=v_{2}^{\infty}+\frac{\partial}{\partial y}\left(C^{\prime} \chi\right)+\frac{\partial}{\partial x}(C \chi)+A^{\prime}\left(y \frac{\partial^{2} \Omega}{\partial y^{2}}-\frac{\partial \Omega}{\partial y}\right)+ \\
& B^{\prime}\left(y \frac{\partial^{2} \Omega}{\partial x \partial y}-\frac{\partial \Omega}{\partial x}\right)+x\left(A \frac{\partial^{2} \Omega}{\partial x \partial y}+B \frac{\partial^{2} \Omega}{\partial y^{2}}\right)
\end{aligned}
$$

where $v_{1}^{\infty}$ and $v_{2}^{\infty}$ are the velocity of the continuum at infinity (boundary conditions) and are substituted from Eq. (7). The symbols $A, A^{\prime}, B, B^{\prime}, C$ and $C^{\prime}$ are defined in Eq. (12). Assuming the incompressibility conditions holds true, one can check the Eq. (A-5) 
satisfies Eq.(5-b). In addition, the hydrostatic stress at the boundary of the ellipse is given by:

$$
\sigma^{h}=p_{\infty}+2 \eta\left(A \frac{\partial^{2} \Omega}{\partial x^{2}}+A^{\prime} \frac{\partial^{2} \Omega}{\partial y^{2}}+\left(B+B^{\prime}\right) \frac{\partial^{2} \Omega}{\partial x \partial y}\right)
$$

where $p_{\infty}$ is the uniform hydrostatic stress far from the elliptical precipitate. 